\title{
Tracing the phase of focused broadband laser pulses
}

\author{
Dominik Hoff $^{1 \star \dagger}$, Michael Krüger ${ }^{2,3 \dagger}$, Lothar Maisenbacher ${ }^{4}$, A. M. Sayler ${ }^{1}$, Gerhard G. Paulus ${ }^{1}$ \\ and Peter Hommelhoff ${ }^{2,4,5 \star}$
}

Precise knowledge of the behaviour of the phase of light in a focused beam is fundamental to understanding and controlling laser-driven processes. More than a hundred years ago, an axial phase anomaly for focused monochromatic light beams was discovered and is now commonly known as the Gouy phase $^{1-4}$. Recent theoretical work has brought into question the validity of applying this monochromatic phase formulation to the broadband pulses becoming ubiquitous today ${ }^{5,6}$. Based on electron backscattering at sharp nanometre-scale metal tips, a method is available to measure light fields with sub-wavelength spatial resolution and sub-optical-cycle time resolution ${ }^{7-9}$. Here we report such a direct, three-dimensional measurement of the spatial dependence of the optical phase of a focused, 4-fs, near-infrared pulsed laser beam. The observed optical phase deviates substantially from the monochromatic Gouy phase-exhibiting a much more complex spatial dependence, both along the propagation axis and in the radial direction. In our measurements, these significant deviations are the rule and not the exception for focused, broadband laser pulses. Therefore, we expect wide ramifications for all broadband lasermatter interactions, such as in high-harmonic and attosecond pulse generation, femtochemistry ${ }^{10}$, ophthalmological optical coherence tomography ${ }^{11,12}$ and light-wave electronics ${ }^{13}$.

In ultrafast light-matter interactions, the phase of the optical carrier field with respect to the pulse envelope's maximum-the carrier-envelope phase (CEP, see Supplementary Information $)^{14}$ is one of the fundamental controls that allows one to steer chemical reactions ${ }^{15}$, the generation of attosecond pulses via high-harmonic generation $^{16}$, and electron emission and acceleration from solid surfaces and nanostructures ${ }^{7,9,17}$, among others. Hence, determining and controlling the CEP is mandatory in many fields using lasers, but taking into account the broadband and often intense and ultrashort nature of these pulses is challenging and an active area of research ${ }^{18,19}$. Further, nonlinear light-matter interactions usually take place in the focus of a beam where the CEP shows a strong spatial dependence. Thus, for a detailed understanding of and field control over these processes, it is essential to take the focal phase evolution, target position, and target extent into account ${ }^{13,20}$. This is as important as controlling the CEP of the input beam itself.

For a focused monochromatic beam, the on-axis phase shift due to diffraction is described by the familiar Gouy phase, which follows a simple arctangent curve ${ }^{1,2}$. However, many of today's coherent light sources, even some as common place as those used in ophthalmological diagnostics, are far from being monochromatic.
Rather, they can span close to, and many even exceed, an octave of spectral bandwidth ${ }^{12,21,22}$. Moreover, recent theoretical studies, based on diffraction theory for pulsed Gaussian beams, yielded spatially dependent phases that significantly deviate from the simple Gouy phase and show a much more complex behaviour that is dictated by the wavelength-dependent input beam geometry $y^{5,6}$. The need for further investigation is underscored by experimental studies, which strongly suggest deviations from the monochromatic Gouy phase $\mathrm{e}^{23-26}$.

In recent years, significant advancements have been made in CEP detection and control ${ }^{14,16,18,27}$, which have facilitated and driven the discovery of more and more processes that are dependent on and can be controlled via the CEP. One such phenomenon is the strong-field, few-cycle-laser-driven backscattering of photoelectrons; namely, those electrons freed by the laser field that can then be driven back to and scattered off their parent matter when the field flips sign within an optical cycle. The large kinetic energy obtained by these electrons strongly depends on the CEP, which we utilize as an experimental signature (see Supplementary Information). This extremely CEPsensitive effect was observed in noble gases and recently also at solid-state nanotips ${ }^{7-9}$. For the latter, strong-field-induced photoemission happens almost exclusively in the enhanced optical nearfield region at the apex of the sharp tip, with a radius of $\sim 10 \mathrm{~nm}$ (ref. 28). Thus, electrons from such a highly localized source are particularly well suited to be used as a sensor to probe focused light fields with resolution better than their natural length and timescales. Namely here, we obtain a spatial resolution of $\sim 10 \mathrm{~nm}$ $(\ll \lambda / 2 \approx 350 \mathrm{~nm}$, the typical length scale of a focus of light with wavelength of $\lambda \approx 700 \mathrm{~nm}$ ), and a CEP resolution of $\sim 80 \mathrm{mrad}$, corresponding to $\sim 60$ as $(\ll 2.3 \mathrm{fs}$, the optical period of light with $\lambda \approx 700 \mathrm{~nm})$.

In this work we present a quantitative, direct, three-dimensional mapping of the CEP evolution of a focused broadband laser beam, spanning a range of seven times the Rayleigh range along the propagation axis and one and a half times the local beam radius perpendicular to the optical axis. To achieve this, we have combined a nanotip-based focus characterization set-up with a xenongas-based, stereographic, above-threshold ionization CEP meter ${ }^{27,29}$. Every laser pulse from a hollow-core fibre compressor is split between the two separate, but synchronized and parallel measurements: one for individual characterization of the random CEP of each shot and the other for recording electron spectra from the laser-nanotip interaction (see Methods and Supplementary Information). In this way, every detected electron from the nanotip can be

\footnotetext{
${ }^{1}$ Helmholtz-Institut Jena and Institut für Optik und Quantenelektronik, Friedrich-Schiller-Universität Jena, Max-Wien-Platz 1, D-07743 Jena, Germany. ${ }^{2}$ Department Physik, Friedrich-Alexander-Universität Erlangen-Nürnberg (FAU), Staudtstr. 1, D-91058 Erlangen, Germany. ${ }^{3}$ Department of Physics of Complex Systems, Weizmann Institute of Science, 234 Herzl St., Rehovot 76100, Israel. ${ }^{4}$ Max-Planck-Institut für Quantenoptik, Hans-Kopfermann-Str. 1,

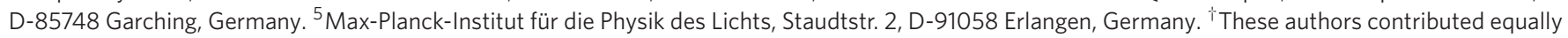
to this work. ^e-mail: dominik.hoff@uni-jena.de; peter.hommelhoff@fau.de
} 
a

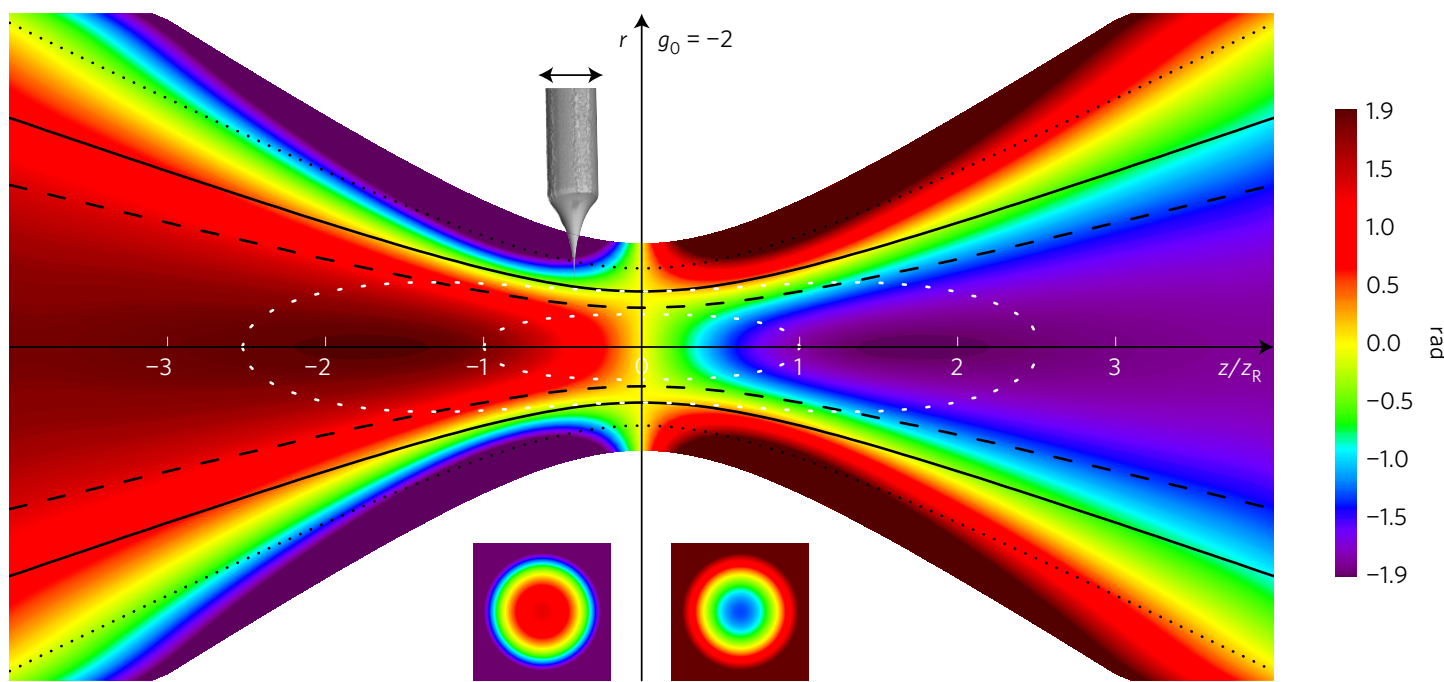

b

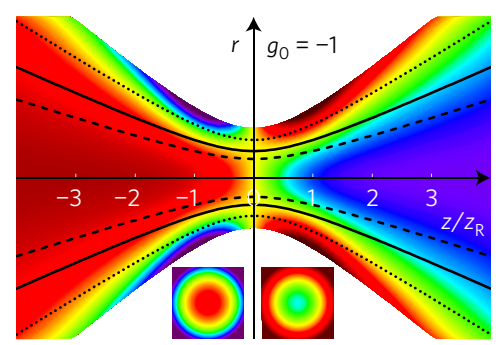

C

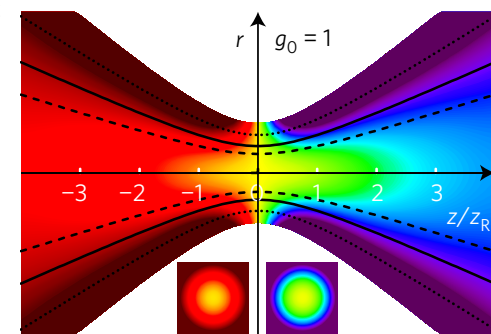

d

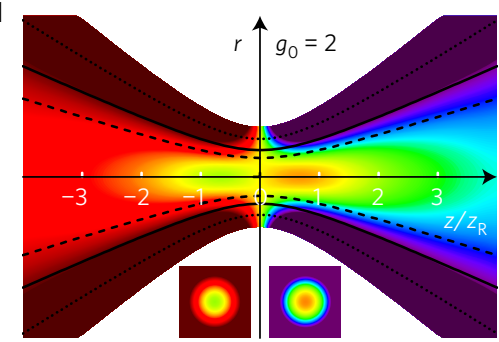

Figure 1 | Carrier-envelope phase in a focused, broadband, pulsed Gaussian beam. a-d, Colour plots showing the calculated CEP in the focus of an input beam with geometry factors of $g_{0}=-2(\mathbf{a}), g_{0}=-1$, that is, isodivergent (b), $g_{0}=1$, which has a wavelength-independent beam waist (c), and $g_{0}=2$ (d) as a function of axial and radial position, $z$ and $r$ respectively. The factor $g_{0}$ is explained in the text and describes the spectral input beam geometry. For visual convenience, the colour is truncated for $|\Delta \phi|>1.9 \mathrm{rad}$ and the beam size at $r>1.86 \mathrm{w}(z)$ where the intensity has dropped below a factor of $10^{-3}$ of the on-axis value. Cross-sections in the $x-y$ plane are given for an alternative perspective of the same data at $z=-0.5$ and $0.5 z_{R}$ in the same radius range (insets). The black lines are hyperbolae related to the local $1 / e^{2}$ intensity radius $w(z)$, namely at $r=w(z) / \sqrt{2}($ dashed), $r=w(z)($ solid $), r=\sqrt{2} w(z)$ (dotted). The white dotted lines in a are isointensity curves of $I_{0} / 2$ and $I_{0} / e^{2}$ (see Supplementary Information). The CEP is probed by recording photoelectron spectra with a metal nanotip on the optical axis and on the hyperbolae in the half-space towards the tip. In the measurements, the Rayleigh length and waist radius are approximately $400 \pm 50 \mu \mathrm{m}$ and $9.0 \pm 0.5 \mu \mathrm{m}$, respectively.

associated with the CEP value for the specific laser pulse producing that electron. The beam towards the nanotip focus measurement was spatially filtered to remove higher transverse modes and provide a good ground state $\left(\mathrm{TEM}_{00}\right)$ approximation as required for the following model. Measurements were done to probe the relative CEP shift both along the laser's propagation axis and radially outward, mapping the cylindrically symmetric three-dimensional space. Both tungsten and gold tips were used. In comparison with previous experimental work ${ }^{23-25}$, in this measurement we probe the focus of a typical intense few-cycle pulse laser beam with a significantly better spatial resolution, scan a larger range of the focal volume and attain a well-defined focus with minimized aberrations by use of an off-axis parabolic mirror and a spatial filter element upfront. In combination with considerable efforts to flatten the spectral phase, these factors allow for a clear and thorough experimental and theoretical investigation of deviations from the monochromatic Gouy phase and additionally reveal the transverse phase structure.

The longitudinal phase difference for a focused, continuous and monochromatic Gaussian beam as compared with a plane wave is described along the propagation axis by

$$
\Delta \psi(z)=-\arctan \left(\frac{z}{z_{\mathrm{R}}}\right)
$$

and is known as the Gouy phase, where $z$ is the laser-propagation direction and $z_{\mathrm{R}}$ is the Rayleigh length ${ }^{1,6,23}$. Laser pulses on the other hand are composed of a coherent superposition of a broad range of wavelengths, leading to a much more complex, spatially dependent phase profile. Moreover, the transverse shape and the divergence of the Gaussian beam in front of the focusing element are, in general, wavelength-dependent. Thus, it is necessary to take into account the wavelength-dependent input beam geometry to determine the spatial dependence of the CEP after the focusing element. A more generalized treatment of the strong focusing of chirp-free pulsed Gaussian beams in terms of their properties prior to focusing combines the concept of enveloped carrier oscillations with fundamental diffraction theor $y^{2}$, which introduces iso-carrierphase fronts and pulse-peak fronts ${ }^{5,6}$. Their difference results in a relative CEP shift in the focal area of

$$
\Delta \phi(z, r)=-\arctan \left(\frac{z}{z_{\mathrm{R}}}\right)+\frac{g_{0}\left[1-2\left(\frac{r}{\mathrm{w}(z)}\right)^{2}\right]}{\frac{z}{z_{\mathrm{R}}}+\frac{z_{\mathrm{R}}}{z}}
$$

where $\Delta \phi$ is defined to be 0 in the focal reference plane (defined by $z=0$ ) for all radii $r$; here, $z_{\mathrm{R}}$ is the Rayleigh length at the centre frequency; $\mathrm{w}(z)$ is the $z$-dependent beam radius, and $r / \mathrm{w}(z)$ is the normalized radial coordinate (see Supplementary Information). The last term of equation (2) describes the difference to the axial Gouy phase, equation (1), and accounts for the wavelengthdependent geometry of the input beam and the difference in curvature of the carrier-phase fronts as compared with that of the pulse fronts. This term scales with $g_{0}-$ a dimensionless geometry 


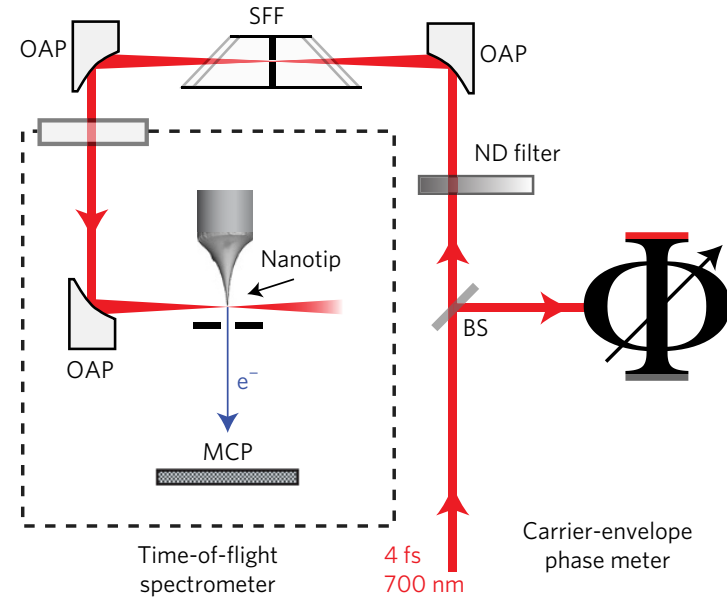

Figure $\mathbf{2}$ | Overview of the phase-tagging scheme with a nanotip. For parallel determination of the laser pulse's CEP and the time of flight of electrons from the nanotip, an amplified $4 \mathrm{fs}, 700 \mathrm{~nm}$ central wavelength, horizontally polarized pulse train from a laser system with a hollow-core fibre compressor is split at the beam splitter (BS) towards the carrier-envelope phase meter arm (right), and the time-of-flight spectrometer arm (left), respectively. The spatial mode of the beam to the nanotip is cleaned with a spatial frequency filter (SFF). The beam is focused inside the ultrahigh-vacuum chamber with a $90^{\circ}$ off-axis parabolic mirror (OAP, $f=50 \mathrm{~mm}$ ) on the nanotip. The time-of-flight spectrometer records the flight time of photoelectrons with a microchannel-plate (MCP) that is then converted into kinetic energy and provides the $x$-axis for the spectrum in Supplementary Fig. 2, see Supplementary Information. The CEP meter measures the randomly varying carrier-envelope phase of each and every shot and thus resolves the spectrum along the $y$ axis in Supplementary Fig. 2. For full details, see Supplementary Information.

factor of the input beam evaluated at the central angular frequency of the laser spectrum $\omega_{0}$, which we call the Porras factor ${ }^{5,6}$.

The Porras factor is given by

$$
g_{0}=\left.\frac{\mathrm{d} Z_{\mathrm{R}}(\omega)}{\mathrm{d} \omega}\right|_{\omega_{0}} \frac{\omega_{0}}{Z_{\mathrm{R}}\left(\omega_{0}\right)}
$$

and represents the normalized first derivative of the input beam's Rayleigh length, $Z_{\mathrm{R}}(\omega)$ (capital letters are used for input beam parameters), with respect to the laser's spectral angular frequencies, $\omega$, evaluated at $\omega_{0}$. The Rayleigh length before the focusing element is linked to the frequency-dependent input beam waist, $\mathrm{W}(\omega)$, by $Z_{\mathrm{R}}(\omega)=\omega \times \mathrm{W}(\omega)^{2} /(2 c)$, where $c$ is the speed of light. Three characteristic cases can be highlighted: $g_{0}=-1$ for an isodiverging input beam, that is, with a constant divergence angle for all $\omega ; g_{0}=0$ for an isodiffracting beam, that is, with a constant Rayleigh length; and $g_{0}=+1$ for a beam with a frequency-independent waist radius ${ }^{6}$ (see Fig. 1 for example phase profiles and Fig. 3 for the case $g_{0}=0$ ). Note that at $g_{0}=0$, all frequencies are diffracted identically in the first-order approximation and hence the phase shift consists merely of the Gouy component. However, one ought not simply assume or expect $g_{0}$ to be 0 as this is just one special and specific case, which is within a continuous range of possible $g_{0}$ values and rarely found in practice.

Figure 3 shows the results of two on-axis measurements together with the monochromatic Gouy phase curve. The measured CEP shows extrema at $z \approx 1.7$ times the Rayleigh length both before and after the focus, resulting in a much steeper slope in the focal plane as compared with the arctangent curve of the Gouy phase. The data can be fitted well by equation (2) at $r=0$, resulting in $g_{0}=-2.1 \pm 0.2$ for the first (I) and $-1.8 \pm 0.3$ for the second (II) data set. These $g_{0}$ values are corroborated by independent measurements of the

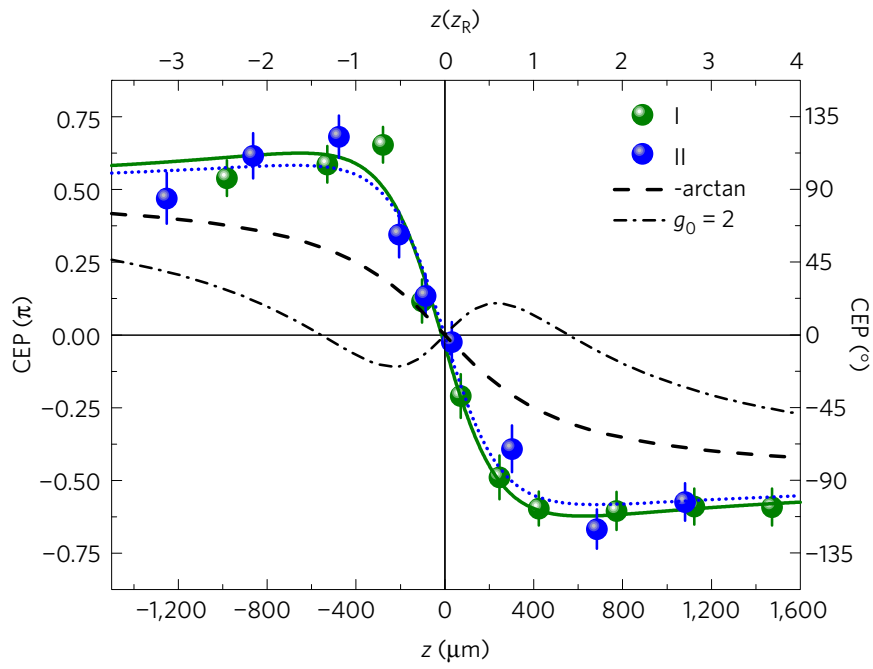

Figure $\mathbf{3}$ | On-axis carrier-envelope phase. CEP as a function of propagation distance, $z$, on the optical axis. Two traces have been recorded, data set I (green spheres, Rayleigh length $z_{R}=380 \pm 50 \mu \mathrm{m}$ ) and II (blue spheres, $\left.z_{R}=365 \pm 50 \mu \mathrm{m}\right)$. The error bars show the measurement uncertainties in CEP of $\approx 0.2 \mathrm{rad}$, as described in the Supplementary Information. The experimental data points were fitted by a theoretical model (equation (2), solid green and dotted blue curves), yielding fitting parameters of $g_{0}=-2.1 \pm 0.2$ (reduced $\chi^{2}=1.3$ ) for data set I and $g_{0}=-1.8 \pm 0.3$ (reduced $\chi^{2}=1.6$ ) for data set II. For comparison, we show the monochromatic Gouy phase, corresponding to the case $g_{0}=0$, for a Rayleigh length of $z_{R}=400 \mu \mathrm{m}$ (black dashed curve), which is clearly unable to explain our observations. The dashed-dotted line depicts the theoretical curve for $g_{0}=+2$ and $z_{R}=400 \mu \mathrm{m}$, as an example phase behaviour for another beam geometry (lineout of Fig. 1d). Data sets I and II have been recorded with different tip materials (see Supplementary Information).

spectral input beam properties outside the vacuum chamber, namely the spectrally resolved Rayleigh length calculated from the beam diameter. This provides an attractive and more easily attainable alternative way to estimate $g_{0}$ via equation (3) and, therefore, obtain an approximate idea of the focal CEP evolution in the interaction region (see Supplementary Information and Fig. 1). Note that further work is warranted and needs to determine whether this technique will fulfil its promise. In addition, the similarity of the experimental curves in Fig. 3 shows the reproducibility of the measurement.

We were also able to produce a three-dimensional map of the CEP by scanning both $z$ and $r$ with the tip (Fig. 4). We observe that there is a strong radial dependence of the CEP, again differing from the monochromatic Gouy phase. Fitting equation (2) to the data points yields $g_{0}=-1.2 \pm 0.3$, where $g_{0}$ and the offsets in $z, r$ and $\Delta \phi$ are free parameters and the Rayleigh length $z_{\mathrm{R}}$ was determined independently by a knife-edge measurement to $z_{\mathrm{R}}=350 \pm 50 \mu \mathrm{m}$ (see Supplementary Information). For negative $g_{0}$ values, as observed in all measurements here, characteristic features of the CEP around the focus can be recognized (see Figs 4 and 1). First, the radial dependence of the CEP surface is concave before, for example, at $z=-800 \mu \mathrm{m}$, and convex after the focus, for example, at $z=800 \mu \mathrm{m}$. Second, radially further out from the axis, that is, $r \gtrsim \mathrm{w}(z)$, the CEP changes sign, implying that the vector potential at the pulse peak changes sign as well (see Supplementary Equation 9). In other words, the CEP evolves with opposite slopes on and off the optical axis. Third, in the focal plane at the waist radius, that is, at $(z, r)=\left(0, \mathrm{w}_{0}\right)$, the phase exhibits a saddle point. In this measurement, the value for $g_{0}$ differs from that obtained previously in the on-axis case as it was done with different laser tuning. This underscores the need to properly characterize the light source in 


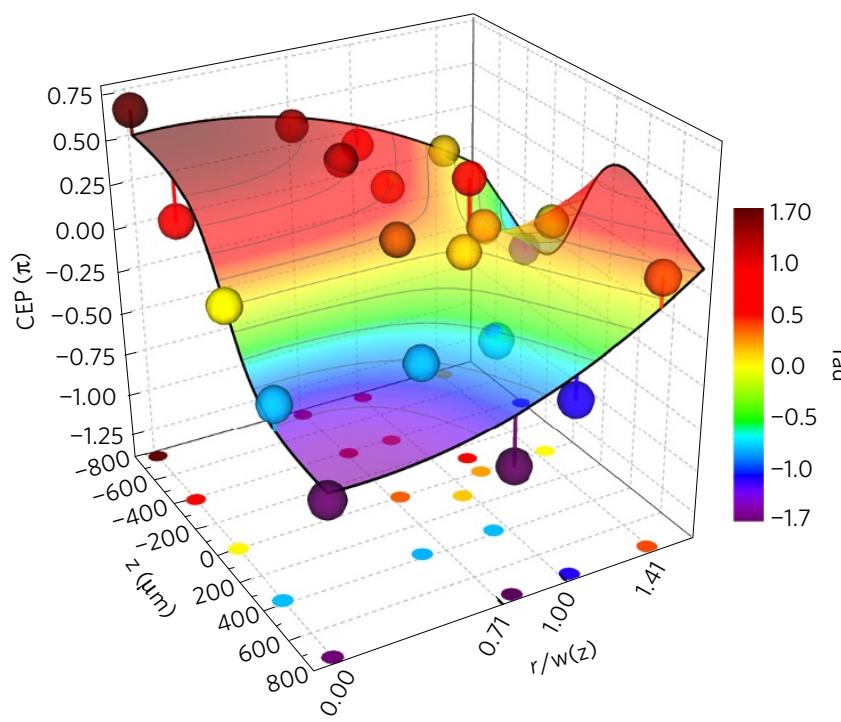

Figure 4 | Off-axis carrier-envelope phase evolution. Three-dimensional plot of the CEP as a function of propagation distance $z$ and normalized radial coordinate $r / w(z)$. The experimental data points are depicted by colour-coded spheres whose size encompasses their uncertainty resulting from measurement errors in positioning and determination of the CEP $(\approx 0.2 \mathrm{rad})$, as described in the Supplementary Information. To further clarify the $z-r$ position of the measured data, each data point is projected in the $z-r$ plane. A surface defined by equation (2) was fitted to the data (coloured), yielding a fit parameter of $g_{0}=-1.2 \pm 0.3$ (reduced $\chi^{2}=3.7$ ). See also Fig. $1 \mathrm{~b}$ for a plot with a similar $g_{0}$ and Supplementary Information for a stereoscopic view and a projected contour plot. The Rayleigh length is $350 \pm 50 \mu \mathrm{m}$, independently determined with a knife-edge measurement.

use and determine the influence of laser tuning parameters, such as hollow-core fibre pressure, on the spectral geometry of the output beam and $g_{0}$ to facilitate advanced phase control in the future.

In the present experiment, the nanotip samples such a small volume of the laser focus in comparison with the Rayleigh range and the focal waist size that it can be assumed that only a single intensity and phase are effective. However, this is not the case in most ultrafast, laser-induced processes. To account for the fact that common targets often experience a large range of laser intensities, one must properly weight the results with the intensity and targetdensity profiles, that is, the intensity-volume effect needs to be taken into account ${ }^{30}$. Further, here we see that this problem is significantly exacerbated for broadband lasers as there is an analogous and coupled phase-volume effect due to the two-dimensional phase profile. In some situations, these complications could be reduced by physically limiting the extent of the target or effectively limiting it by selecting a high-order process. However, they are by no means eliminated. For example, even for a target, very thin in the laser-propagation direction, $\Delta z \ll z_{\mathrm{R}}$, the phase is often strongly radially dependent even within the beam width. Thus, the common assumption of an arctangent dependence of the CEP is often unwarranted and insufficient.

Although these effects can complicate interpretation, they also have the potential to be used to enhance desired effects and perhaps even be utilized in novel ways, for instance, to improve phase matching in high-order harmonic and attosecond pulse generation. Further, one could optimize these effects by tailoring the interaction region via the input beam geometry, expressed by $g_{0}$. In addition, we expect that recent developments such as particle trapping and acceleration of atoms with femtosecond laser pulses may benefit from the CEP control demonstrated here through an interesting well-like structure formed by the CEP gradients ${ }^{31-33}$. Hence, new ways of controlling atoms with large forces through ultrashort, strong laser fields may result. The detailed knowledge of the phase evolution found here impacts many fields where knowledge and control of the optical phase is vital and fascinating developments can be foreseen.

\section{Methods}

Methods, including statements of data availability and any associated accession codes and references, are available in the online version of this paper.

Received 10 January 2017; accepted 23 May 2017; published online 10 July 2017

\section{References}

1. Gouy, L. G. Sur une propriété nouvelle des ondes lumineuses. C. R. Acad. Sci. Paris 110, 1251-1253 (1890).

2. Born, M. \& Wolf, E. Principles of Optics: Electromagnetic Theory of Propagation, Interference and Diffraction of Light (Cambridge Univ. Press, 1999).

3. Siegman, A. E. Lasers Vol. 37 (University Science Books, 1986).

4. Visser, T. \& Wolf, E. The origin of the Gouy phase anomaly and its generalization to astigmatic wavefields. Opt. Commun. 283, 3371-3375 (2010).

5. Porras, M. A. Diffraction effects in few-cycle optical pulses. Phys. Rev. E 65, 026606 (2002).

6. Porras, M. A. Characterization of the electric field of focused pulsed Gaussian beams for phase-sensitive interactions with matter. Opt. Lett. 34, 1546-1548 (2009).

7. Krüger, M., Schenk, M. \& Hommelhoff, P. Attosecond control of electrons emitted from a nanoscale metal tip. Nature 475, 78-81 (2011).

8. Wachter, G. et al. Electron rescattering at metal nanotips induced by ultrashort laser pulses. Phys. Rev. B 86, 035402 (2012).

9. Piglosiewicz, B. et al. Carrier-envelope phase effects on the strong-field photoemission of electrons from metallic nanostructures. Nat. Photon. 8, 37-42 (2014).

10. Gordon, R. J. \& Barge, V. J. Effect of the Gouy phase on the coherent phase control of chemical reactions. J. Chem. Phys. 127, 204302 (2007).

11. Johnson, J. L., Dorney, T. D. \& Mittleman, D. M. Enhanced depth resolution in terahertz imaging using phase-shift interferometry. Appl. Phys. Lett. 78, 835-837 (2001).

12. Drexler, W. \& Fujimoto, J. G. Optical Coherence Tomography: Technology and Applications (Springer Science \& Business Media, 2008).

13. Krausz, F. \& Stockman, M. I. Attosecond metrology: from electron capture to future signal processing. Nat. Photon. 8, 205-213 (2014).

14. Udem, T., Holzwarth, R. \& Hänsch, T. W. Optical frequency metrology. Nature 416, 233-237 (2002)

15. Kling, M. et al. Control of electron localization in molecular dissociation. Science 312, 246-248 (2006).

16. Baltuška, A. et al. Attosecond control of electronic processes by intense light fields. Nature 421, 611-615 (2003).

17. Zherebtsov, S. et al. Controlled near-field enhanced electron acceleration from dielectric nanospheres with intense few-cycle laser fields. Nat. Phys. 7, 656-662 (2011).

18. Fordell, T., Miranda, M., Arnold, C. \& L'Huillier, A. High-speed carrier-envelope phase drift detection of amplified laser pulses. Opt. Exp. 19, 23652-23657 (2011).

19. Paasch-Colberg, T. et al. Solid-state light-phase detector. Nat. Photon. 8 , 214-218 (2014).

20. Goulielmakis, E. et al. Attosecond control and measurement: lightwave electronics. Science 317, 769-775 (2007).

21. Shverdin, M., Walker, D., Yavuz, D., Yin, G.-Y. \& Harris, S. E. Generation of a single-cycle optical pulse. Phys. Rev. Lett. 94, 033904 (2005).

22. Wirth, A. et al. Synthesized light transients. Science 334, 195-200 (2011).

23. Lindner, F. et al. Gouy phase shift for few-cycle laser pulses. Phys. Rev. Lett. 92, 113001 (2004).

24. Tritschler, T., Hof, K., Klein, M. \& Wegener, M. Variation of the carrier-envelope phase of few-cycle laser pulses owing to the Gouy phase: a solid-state-based measurement. Opt. Lett. 30, 753-755 (2005).

25. Major, B., Nemes, D., Porras, M. A., Horváth, Z. L. \& Kovács, A. P. Carrier-envelope phase changes in the focal region: propagation effects measured by spectral interferometry. Appl. Opt. 54, 10717-10724 (2015).

26. Schlaepfer, F. et al. Gouy phase shift for annular beam profiles in attosecond experiments. Opt. Express 25, 3646 (2017).

27. Wittmann, T. et al. Single-shot carrier-envelope phase measurement of few-cycle laser pulses. Nat. Phys. 5, 357-362 (2009).

28. Thomas, S., Krüger, M., Förster, M., Schenk, M. \& Hommelhoff, P. Probing of optical near-fields by electron rescattering on the $1 \mathrm{~nm}$ scale. Nano Lett. 13, 4790-4794 (2013). 
29. Johnson, N. G. et al. Single-shot carrier-envelope-phase-tagged ion-momentum imaging of nonsequential double ionization of argon in intense 4-fs laser fields. Phys. Rev. A 83, 013412 (2011).

30. Sayler, A., Wang, P., Carnes, K. \& Ben-Itzhak, I. Determining intensity dependence of ultrashort laser processes through focus z-scanning intensity-difference spectra: application to laser-induced dissociation of $\mathrm{H} 2+$ J. Phys. B: At. Mol. Opt. Phys. 40, 4367-4378 (2007).

31. Shane, J. C., Mazilu, M., Lee, W. M. \& Dholakia, K. Effect of pulse temporal shape on optical trapping and impulse transfer using ultrashort pulsed lasers. Opt. Express 18, 7554-7568 (2010).

32. Freegarde, T., Walz, J. \& Hänsch, T. Confinement and manipulation of atoms using short laser pulses. Opt. Commun. 117, 262-267 (1995).

33. Eichmann, U., Nubbemeyer, T., Rottke, H. \& Sandner, W. Acceleration of neutral atoms in strong short-pulse laser fields. Nature 461, 1261-1264 (2009).

\section{Acknowledgements}

We gratefully acknowledge M. Förster for supplying nanotips, and P. Dombi and

$\mathrm{S}$. Thomas for support in the measurement campaign. This work has been supported by the DFG Grants PA 730/5 and SPP-1840 QUTIF, Laserlab-Europe EU-H2020 654148, ERC Grant NearFieldAtto, and the DFG Cluster of Excellence Munich Center for Advanced Photonics. D.H. acknowledges the Helmholtz Association for financial support. M.K. acknowledges the Minerva Foundation and the Koshland Foundation for financial support.

\section{Author contributions}

All authors contributed to all parts of the experiment including the final version of the manuscript.

\section{Additional information}

Supplementary information is available in the online version of the paper. Reprints and permissions information is available online at www.nature.com/reprints. Publisher's note: Springer Nature remains neutral with regard to jurisdictional claims in published maps and institutional affiliations. Correspondence and requests for materials should be addressed to $\mathrm{D} . \mathrm{H}$. or $\mathrm{PH}$.

\section{Competing financial interests}

The authors declare no competing financial interests. 


\section{Methods}

We employ a phase-tagging method ${ }^{29}$ for recording the CEP-dependent electron backscattering at tungsten and gold nanotips, placed at different points near the focus (see Supplementary Figs 1 and 2). This method utilizes simultaneous measurements of electron time-of-flight spectra from the metal nanotip in event mode on the one hand, and of the CEP of every single shot of the few-cycle pulse train on the other, the latter being determined by a phasemeter ${ }^{27}$. The events in both measurements are synchronized by triggering on the same laser pulse. Both measurements rely on the strong CEP dependence of backscattered, laser-driven photoelectrons; namely, those electrons that return to their parent matter and scatter elastically back from it after acceleration in the optical field.
In the CEP meter the two electron spectra that build up from xenon in the left and right direction of the horizontal laser polarization are recorded and compared by calculating the asymmetry parameter $A=\left(N_{\mathrm{L}}-N_{\mathrm{R}}\right) /\left(N_{\mathrm{L}}+N_{\mathrm{R}}\right)$, that is, the contrast, where $N$ is the electron yield left and right, respectively. From this parameter, the CEP of the laser shot can be evaluated ${ }^{27}$. Exploiting the high spatial resolution and pronounced CEP sensitivity of the electron backscattering at the metal nanotip, we recorded phase-tagged kinetic-energy spectra at positions on the optical axis and off-axis on hyperbolic curves in a plane along the focus (see Fig. 1 and Supplementary Information 2).

Data availability. The data that support the plots within this paper and other findings of this study are available from the corresponding author on reasonable request. 